\title{
Synthesis of a pentathiophene fluorescent probe, $4^{\prime}, 3^{\prime \prime}$-bis-carboxymethyl- $\left[2,2^{\prime} ; 5^{\prime}, 2^{\prime \prime} ; 5^{\prime \prime}, 2^{m} ; 5^{\prime \prime}, 2^{m}{ }^{m}\right]$ quinquethiophene-5,5'
}

Andreas Åslund

Linköping University

K. Peter Nilsson

Linköping University

Peter Konradsson

Linköping University

\section{Method Article}

Keywords: fluorescent probe, amyloid, prion, synthesis, oligothiophene

Posted Date: January 26th, 2010

DOI: https://doi.org/10.1038/nprot.2010.24

License: (1) (i) This work is licensed under a Creative Commons Attribution 4.0 International License.

Read Full License 


\section{Abstract}

\section{Introduction}

Poly and oligo-thiophenes have previously been used for _in vitro_ ${ }^{1,2}$,_ex vivo_ ${ }_{-}^{3,4}$ and _in vivo_ ${ }^{5}$ imaging of protein aggregates. The probe $\backslash(\mathrm{p}-\mathrm{FTAA})$ has been developed for the purpose of in vivo staining of protein aggregates such as amyloid deposits. It effectively passes the blood brain barrier and imaging can be performed live with two-photon imaging or _ex vivo_ ${ }^{5}$. The straightforward synthesis of p-FTAA, including two Suzuki couplings, makes it an attractive probe for studies of most diseases involving protein aggregates.

\section{Reagents}

- 3-Thiopheneacetic acid \(Sigma-Aldrich, cat. no. 220639) - Acetyl chloride \(Sigma-Aldrich, cat. no. 00990) - Methanol \(Merck, cat. no. 1.08325) - N-bromosuccinimide \(NBS) \(Sigma-Aldrich, cat. no. B81255) - Chloroform \(Prolabo, cat. no. 83626.320) - Acetic acid \(Sigma-Aldrich, cat. no. A6283) - Silica Gel for flush column chromatograpy \(Sigma-Aldrich, cat. no. 22716-6) - 2,5-Thiophenediboronic acid \ (Sigma-Aldrich, cat. no. 470317) - PEPPSI-Ipr® \(\[1,3-Bis \(2,6-Diisopropylphenyl)imidazol-2-ylidene] $\backslash(3-$ chloropyridyl)palladium (II) dichloride) \(Sigma-Aldrich, cat. no. 669032) - Potassiumcarbonate \(SigmaAldrich, cat. no. 20961-9) - Toluene \(Merck, cat. no. 1.08325) - 5-\(Dihydroxyboryl)-2-thiophenecarboxylic acid $\backslash$ (Frontier Scientific, cat. no. C1695) - Sodiumhydroxide $\backslash($ Sigma-Aldrich, cat. no. 45291-2) Deuterated chloroform $\backslash($ Sigma-Aldrich, cat. no. 151823) - Deuterated DMSO \(Sigma-Aldrich, cat. no. 151874) - Deuterated D2O \(Sigma-Aldrich, cat. no. 151882) - Thin-layer chromatography plates on glas backing, silica gel 60 F254 \(Merck) - Ethylacetate \(Prolabo cat. no. 23882.321) - Celite ${ }^{\circledR} 545 \backslash$ (Prolabo cat. no. 22552.290) - Trifluoroacetic acid \(Merck cat. no. 8.08260) - Diethylether \(Prolabo cat. no. 23811.292) - HCl \(Scharlau cat. no. AC0741) - Dioxane \(Prolabo cat. no. 83620.320)

\section{Equipment}

- Magnetic hotplate stirrer - Digital temperature probe - Oil bath - 100 and $250 \mathrm{~mL}$ round-bottom flasks Water condenser $\backslash$ (to fit neck of flask) - Teflon-coated magnetic stirrer bar - Heat gun - Rotary evaporator $\backslash$ (Büchi) - Pyrex chromatographic column $\backslash$ (approx. diameter $3 \mathrm{~cm}$ ) - NMR tubes - VersaFlash ${ }^{\circledR}$ purification system with reversed phase columns $\backslash($ Sigma-Aldrich)

\section{Procedure}

**Synthesis of $\backslash(2$-bromo-thiophen-3-yl)-acetic Acid methyl ester $\backslash(2) \star * 1]$ To a solution at $0{ }^{\circ} \mathrm{C}$ with 3thiopheneacetic acid $\backslash(1.42 \mathrm{~g}, 10 \mathrm{mmol})$ and methanol $\backslash(20 \mathrm{~mL})$ add acetic acid $\backslash(1 \mathrm{~mL})$ slowly. 2] Dilute with toluene and wash with $\mathrm{NaHCO} \backslash$ (sat. aq.) until basic. 3] Dry with $\mathrm{MgSO}_{4}$, filter and concentrate. 4] Dissolve in $20 \mathrm{~mL}$ chloroform/acetic acid $\backslash(1: 1)$ and cool to $0{ }^{\circ} \mathrm{C}$. 5] Add $1.78 \mathrm{~g} \mathrm{NBS} \backslash(10 \mathrm{mmol}) 6$ ] After five hours dilute with toluene and wash with $\mathrm{NaHCO}_{3} \backslash$ (sat. aq.) until basic. 7] Dry with $\mathrm{MgSO}_{4}$, filter and 
concentrate. 8] Purify on flush column chromatography $\backslash$ (toluene). **Synthesis of $\backslash(3 "-$

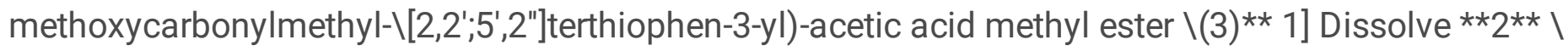
$(2.35 \mathrm{~g} 10 \mathrm{mmol})$ and 2,5-thiophenediboronic acid $\backslash(0.781 \mathrm{~g}, 4.5 \mathrm{mmol})$ in $20 \mathrm{~mL}$ degassed toluene/methanol $\backslash(1: 1)$. 2] Add $4.14 \mathrm{~g} \backslash(30 \mathrm{mmol}) \mathrm{K}_{2} \mathrm{CO}_{3}$ and PEPPSI-Ipr $\backslash(0.204 \mathrm{~g}, 0.30 \mathrm{mmol})$ to the solution and heat at $80{ }^{\circ} \mathrm{C}$ for 10 minutes. 3] Filter the reaction mixture on celite, dilute with toluene and wash with $\mathrm{NaHCO}_{3} \backslash$ (sat. aq.). 4] Dry with $\mathrm{MgSO}_{4}$, filter and concentrate. 5] Purify the crude reaction mixture on a reversed phase column $\backslash$ (methanol $/ \mathrm{H}_{2} \mathrm{O} 4: 1,0.05 \%$ trifluoroacetic acid) to give the product. **Synthesis of $\backslash\left(5,5^{\prime \prime}\right.$-dibromo-3"-methoxycarbonylmethyl- $\left.\backslash 2,2^{\prime} ; 5^{\prime}, 2^{\prime \prime}\right]$ terthiophen 3-yl)-acetic acid methyl ester $\backslash(4) \star \star ~ 1] ~ A d d ~ N B S ~ \(0.460 \mathrm{~g}, 2.04 \mathrm{mmol})$ to a solution of the trimer $\backslash\left(\star \star 3^{\star \star}, 0.382 \mathrm{~g}, 0.973 \mathrm{mmol}\right)$ in chloroform/acetic acid $\backslash\left(1: 1,5 \mathrm{~mL}, 0^{\circ} \mathrm{C}\right)$. 2] Stir for 4 hours before dilution with diethyl ether $\left.\backslash(20 \mathrm{~mL}) 3\right]$ Wash with $\mathrm{NaHCO}_{3} \backslash$ (sat. aq., $\left.20 \mathrm{~mL}\right)$ and finally water $\backslash(20 \mathrm{~mL})$. 4] Dry with $\mathrm{MgSO}_{4}$, filter and concentrate. 5] Purify by flash column chromatography $\backslash($ toluene). **Synthesis of 4',3"'-bis-

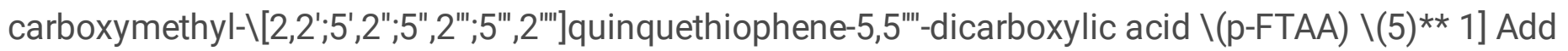
$\mathrm{K}_{2} \mathrm{CO}_{3} \backslash(4.14 \mathrm{~g}, 30 \mathrm{mmol}), \operatorname{PEPPSI}-\mathrm{IPr} \backslash(0.204 \mathrm{~g}, 0.30 \mathrm{mmol})$ and 5- $\backslash($ dihydroxyboryl)-2thiophenecarboxylic acid to a solution of $* \star * \star \star ~ \backslash(5.50 \mathrm{~g}, 10 \mathrm{mmol})$ in degassed toluene/methanole $\backslash(1: 1$, $30 \mathrm{~mL})$. 2] Heat at $80^{\circ} \mathrm{C}$ for 10 minutes 3] Add $\mathrm{HCl} \backslash(20 \mathrm{~mL}, 1 \mathrm{M}$, aq. $)$ and collect the red precipitate 4] Dissolve the precipitate in boiling dioxane and filter it on celite followed by precipitation by addition of a small amount of water. 5] Dry the precipitate and add two equimolar amounts of $\mathrm{NaOH} \backslash(1 \mathrm{M}$, aq. $)$ 6] The product is afforded by concentration or freeze drying.

\section{Timing}

One week

\section{Critical Steps}

During the cross coupling reactions generating product $* \star 5^{\star *}$ and $* * 3 * \star$ both temperature and time should be precisely monitored. If available a microwave reactor can be used. The purification of product 3 should be performed on reversed phase column for best result.

\section{Troubleshooting}

Low yield: Repeat reaction with fresh reagents.

\section{Anticipated Results}

**Product $2 * \star$ Typical isolated yield of ${ }^{* *} 2^{\star *}$ should be $2.16 \mathrm{~g} \backslash(92 \%)$. Analytical data: ${ }^{* *}{ }^{1} \mathrm{H}-\mathrm{NMR} \mathrm{R}^{\star \star} \backslash$ $(\mathrm{CDCl} 3)$ ?: $3.63 \backslash(\mathrm{s}, 2 \mathrm{H}), 3.71 \backslash(\mathrm{s}, 3 \mathrm{H}), 6.30 \backslash(\mathrm{d}, 1 \mathrm{H}, \mathrm{J}=5.7 \mathrm{~Hz}), 7.33 \backslash(\mathrm{d}, 1 \mathrm{H}, \mathrm{J}=5.7 \mathrm{~Hz}){ }^{* * 13} \mathrm{C}-\mathrm{NMR}$ ** $\backslash$

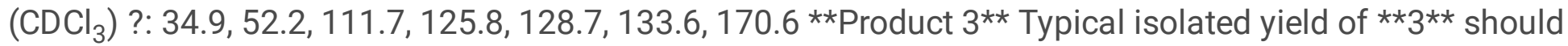
be $1.50 \mathrm{~g} \backslash(85 \%)$ as slightly yellow colored oil. Analytical data: ${ }^{*}{ }^{1} \mathrm{H}^{-N_{M R}}{ }^{\star \star} \backslash\left(\mathrm{CDCl}_{3}\right)$ ?: $3.72 \backslash(\mathrm{s}, 4 \mathrm{H}), 3.79$ 
$\backslash(\mathrm{s}, 6 \mathrm{H}), 7.05 \backslash(\mathrm{d}, 2 \mathrm{H}, \mathrm{J}=5.2 \mathrm{~Hz}), 7.14 \backslash(\mathrm{s}, 2 \mathrm{H}), 7.25 \backslash(\mathrm{d}, 2 \mathrm{H}, \mathrm{J}=5.2 \mathrm{~Hz}) ;{ }^{*}{ }^{13} \mathrm{C}-\mathrm{NMR}{ }^{\star \star} \backslash(\mathrm{CDCl})$ ?: 34.7 , $52.3,124.9,127.5,130.5,130.6,133.0,135.9,171.5 ;{ }^{*} \mathrm{HRMS}^{* *} \_\mathrm{m} / \mathrm{z}_{-}$calcd. for $\mathrm{C}_{18} \mathrm{H}_{16} \mathrm{O}_{4} \mathrm{~S}_{3}: \backslash[\mathrm{M}]+$ 392.0211; Found: 392.0228. **Product $4 * *$ Typical isolated yield of $* \star 4 * \star$ should be $0.482 \mathrm{~g} \backslash(90 \%)$. Analytical data: ${ }^{* *}{ }^{1} \mathrm{H}-\mathrm{NMR}^{\star \star} \backslash\left(\mathrm{CDCl}_{3}\right)$ ?: $3.70 \backslash(\mathrm{s}, 4 \mathrm{H}), 3.73 \backslash(\mathrm{s}, 6 \mathrm{H}), 7.02 \backslash(\mathrm{s}, 2 \mathrm{H}), 7.08 \backslash(\mathrm{s}, 2 \mathrm{H}) ;{ }^{*}{ }^{13} \mathrm{C}-$ $\mathrm{NMR}^{\star \star} \backslash(\mathrm{CDCl} 3)$ ?: 34.5, 52.4, 111.8, 127.2, 127.8, 131.1, 134.1, 134.8, 170.8**Product 5** Typical isolated yield of $* \star 5^{\star \star}$ should be $6.00 \mathrm{~g} \backslash(85 \%)$ as red powder. Analytical data: ${ }^{* *}{ }^{1} \mathrm{H}-\mathrm{NMR}^{\star \star} \backslash(\mathrm{DMSO}-\mathrm{D} 6)$ ?: $3.76 \backslash(\mathrm{s}, 4 \mathrm{H}), 7.32 \backslash(\mathrm{s}, 2 \mathrm{H}), 7.38 \backslash(\mathrm{d}, 2 \mathrm{H}, \mathrm{J}=4.10 \mathrm{~Hz}), 7.45 \backslash(\mathrm{s}, 2 \mathrm{H}), 7.66 \backslash(\mathrm{d}, 2 \mathrm{H}, \mathrm{J}=4.10 \mathrm{~Hz}) ;{ }^{* * 13} \mathrm{C}^{\star *}$ $\mathrm{NMR} \backslash\left(\mathrm{D}_{2} \mathrm{O}, 45^{\circ} \mathrm{C}\right)$ ?: $38.4,124.4,126.6,129.1,131.9,132.0,134.4,134.7,135.5,139.6,140.9,169.9$, 179.4; **HRMS**_m/z_calcd for $\mathrm{C}_{26} \mathrm{H}_{15} \mathrm{O}_{8} \mathrm{~S}_{5}: \backslash[\mathrm{M}-\mathrm{H}]-614.9371$; Found 614.9422

\section{References}

1. Nilsson, K.P.R., Herland, A., Hammarström, P. \& Inganäs, O. Conjugated polyelectrolytes: Conformationsensitive optical probes for detection of amyloid fibril formation. _Biochemistry_ ${ }^{* \star} 44^{\star \star}, 3718-3724 \backslash$ (2005). 2. Åslund, A. et al. Studies of luminescent conjugated polythiophene derivatives: enhanced spectral discrimination of protein conformational States. _Bioconjug. Chem._ ${ }^{\star *} 18{ }^{\star *}, 1860-8 \backslash(2007) .3$. Nilsson, K.P.R. et al. Imaging Distinct Conformational States of Amyloid-beta Fibrils in Alzheimer's Disease Using Novel Luminescent Probes. _ACS Chem. Biol._ ${ }^{\star} 2 \star \star, ~ 553-560 \backslash(2007)$. 4. Sigurdson, C.J. et al. Prion strain discrimination using luminescent conjugated polymers. _Nature Methods_ ${ }^{\star \star} 4 * \star, 1023-30$ $\backslash(2007)$. 5. Åslund, A. et al. Novel pentameric thiophene derivatives for in vitro and in vivo optical imaging of a plethora of protein aggregates in cerebral amyloidoses. _ACS Chem. Biol._ ${ }^{\star \star 4 * *}, 673-84 \backslash(2009)$.

\section{Acknowledgements}

Our work is supported by the Swedish Foundation for Strategic Research \(KPRN, PK and AÅ), the Knut and Alice Wallenberg foundation \(KPRN), and a grant from the European Union FP-7: "LUPAS" \(KPRN).

\section{Figures}

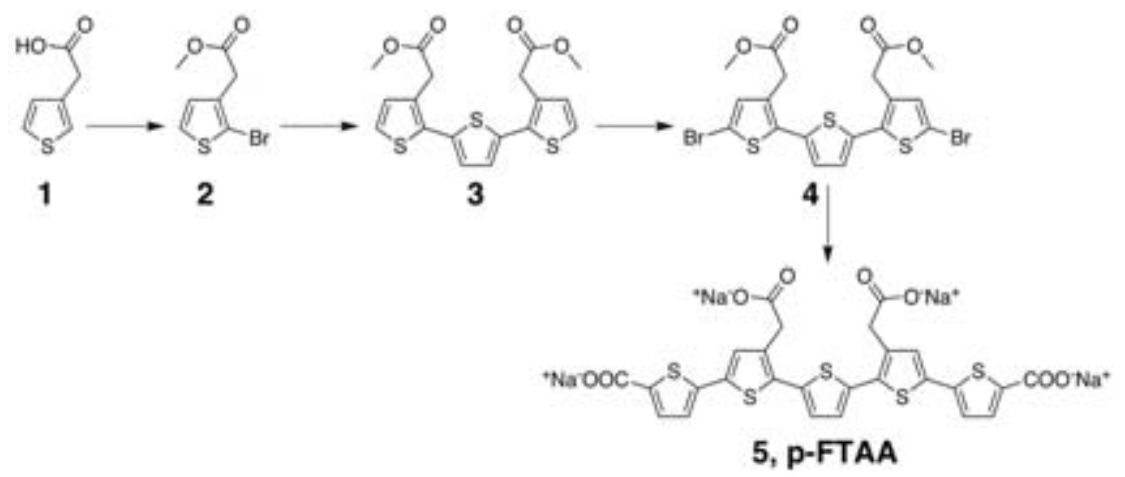

Figure 1 
*Scheme 1.* Proposed synthetic route to p-FTAA (5)

Page 5/5 